\title{
Microvascular Architecture of the Rabbit Eye: A Scanning Electron Microscopic Study of Vascular Corrosion Casts
}

\author{
Hiroyoshi NINOMIYA ${ }^{1)}$, Tomo INOMATA ${ }^{1)}$ and Nobuyuki KANEMAKI ${ }^{2)}$ \\ ${ }^{1)}$ Departments of Laboratory Animal Science and ${ }^{2)}$ Veterinary Internal Medicine III, Azabu University, 1-17-71 Fuchinobe Sagamihara, \\ 229-8501, Japan
}

(Received 30 October 2007/Accepted 1 May 2008)

\begin{abstract}
The microvasculature of the eyes of 5 rabbits was investigated using scanning electron microscopy on corrosion casts. The study revealed that the pars plana vessels draining blood from the iris and ciliary body coursed directly into the anterior vortex venous system constituting the scleral venous plexus (the venous circle of Hovius). The episcleral vasculature was found to possess a specialized morphology, with channels draining the aqueous humor. The capillaries of the third palpebral, bulbar and palpebral conjunctiva formed a single-layered capillary network approximately parallel to the epithelium and formed a well-developed venous plexus in the stroma. The retina was found to be merangiotic, meaning that vessels were present only in a small part of the retina, extending in a horizontal direction to form bands on either side of the optic disc. Channels representing the aqueous veins that drained blood mixed with aqueous humor were found to derive directly from the suprachoroidal space and communicate with the scleral venous plexus via the anterior vortex veins. The functional significance of the microvasculature of the iris, cilia, retina and choroid is discussed in this report as well. The elaborate microvasculature of the conjunctiva may be a prerequisite for the exchange of nutrients and gasses between the cornea and the vessels across the conjunctival epithelium when the eyelids are shut during sleep, and possibly for the dynamics of eye drop delivery. The scleral venous plexus in rabbits may be analogous to the scleral venous sinus (Schlemm's canal) in rats, primates and humans. KEY WORDS: corrosion casts, eye, microvasculature, rabbit, SEM.
\end{abstract}

J. Vet. Med. Sci. 70(9): 887-892, 2008

The rabbit has undoubtedly been one of the animals most commonly used in ophthalmology-related biomedical experiments. The general distribution of the major vessels in the rabbit eyeball has already been outlined $[5,11,12$, 20]. Scanning electron microscopy (SEM) has been used to study corrosion casts, and comparative descriptions of the rabbit's ocular vasculature have been published $[2,5,8,11$, 12]. These studies indicated marked interspecies variation in vasculature among mammalian eyes. The retinal vasculature of the rabbit is merangiotic, meaning that the vessels extend partially to either side of the optic disc [23]. For the rabbit, the data currently available on the anterior eye segment, retina, choroids and aqueous drainage route are incomplete, poorly documented, and often unclear. Although the evaluation of vascular changes in the retinal region is of diagnostic importance in assessing the irritancy of the ocular surface, no definitive description of the pattern of capillary organization in this region has yet been reported. Solid morphological knowledge of the retinal angioarchitecture is an absolute requisite for the understanding and utilization of ophthalmologic and fluorangiographic observations.

To further study the microvascular architecture of the rabbit's eye, we have performed a detailed SEM on corrosion casts of rabbit ocular vessels.

\footnotetext{
* Correspondence to: Ninomiya, H., Department of Laboratory Animal Science Azabu University, 1-17-71 Fuchinobe Sagamihara, 229-8501, Japan.

e-mail: ninomiya@azabu-u.ac.jp
}

\section{MATERIALS AND METHODS}

Ten eyes from New Zealand White rabbits $(3.5-4.0 \mathrm{~kg}$ in weight, adult, male) were used in this study.

Ophthalmoscopy: The animals were anesthetized with sodium pentobarbital $(20 \mathrm{mg} / \mathrm{kg})$ injected peritoneally, and their pupils were dilated with an instillation of $0.5 \%$ tropicamide and $0.5 \%$ phenylephrine eyedrops for ophthalmoscopy. The fundi were examined under an indirect ophthalmoscope, and photographed using a fundic camera (Kowa RC-11, Kowa Co., Ltd., Tokyo, Japan).

Corrosion casts: After ophthalmic examination, the carotid artery of each rabbit was opened to bleed the animal. The jugular vein was also opened, a catheter was introduced into the carotid artery, and the rabbit was perfused with a physiological saline solution warmed to $37^{\circ} \mathrm{C}$ via this catheter using a syringe under manual pressure. Perfusion continued until the saline solution emerging from the jugular vein was completely clear of blood. The orbital blood vessels were manually injected from the right and left carotid arteries. Approximately $10 \mathrm{~m} l$ of a combination of methylmethacrylate monomer and Mercox (Dainippon Ink \& Chemical Co., Ltd., Tokyo, Japan) (ratio in volume=7:3) was injected under an injection pressure of 100 to 120 $\mathrm{mmHg}$. After polymerization, the eye was macerated for $1-$ 2 days via repeated baths in a $20 \% \mathrm{NaOH}$ solution at $50^{\circ} \mathrm{C}$, and the samples were then rinsed in running water. Under a dissecting microscope, each part of the eye was isolated, mounted on an aluminum stub, sputtered with gold in an ion-coater (IB-3, Eiko Engineering Co., Ltd., Ibaraki, Japan) and examined under a scanning electron microscope 
(ABT-32, Topcon Co., Ltd., Tokyo, Japan).

Histology: Another rabbit was used to provide a histological reference for our casts. The animal was treated with the above mentioned procedure. Its tissue was fixed with $10 \%$ formalin, dehydrated in ethanol and embedded in paraffin wax. Afterwards, 5- $\mu \mathrm{m}$ sections were cut and stained with hematoxylin and eosin.

All animals were treated in accordance with the Azabu University Animal Care and Use Guidelines and the ARVO Statement for the Use of Animals in Ophthalmic and Visual Research. The animal facility at Azabu University is accredited by the Office of Laboratory Animal Welfare (OLAW) (\#A5393-01), U.S.A.

\section{RESULTS}

The eyeball of the rabbit was found to receive most of its blood supply from the long posterior ciliary, short posterior ciliary, anterior ciliary arteries and chorioretinal arteries that is a resultant anastomosis of the central retinal, long posterior ciliary, and short posterior ciliary arteries (Fig. 1).

Vasculature of the anterior segment of the eyeball: Blood is supplied to the ciliary process by the iridociliary ring artery, which originates from the long posterior ciliary arteries. The ring artery travels about halfway between the iris and the lateral border of the eyeball, where the artery gives rise to the capillary beds of the ciliary process (Fig. 2A). The microvessels in the ciliary process form radiallyarranged, closely-packed plates of vessels (Fig. 2B), consisting of the marginal capillaries, the intraprocess capillaries and the collecting venules. The marginal capillaries (21.0-27.5 $\mu \mathrm{m}$ in diameter), exhibit an irregular bore with alternating expansions. They directly connect the ciliary process arterioles with the collecting venules, forming a 'thoroughfare channel'. The capillaries in the ciliary process are thin (10.0-11.8 $\mu \mathrm{m}$ in diameter), and some are connected with adjacent ciliary processes. These capillaries drain into the collecting venules and exhibit less undulation and a smaller diameter. The ciliary collecting venules empty into the pars plana venules (Fig. 2B). The pars plana venules then drain into the large veins of the anterior vortex system. The anterior and posterior vortex veins draining the choroidal veins travel toward the sclerocorneal junction region and form an incomplete circle called the scleral venous plexus (Fig. 3).

The blood supply of the iris arises primarily from the iridociliary ring artery, which is augmented by the anterior ciliary arteries. The branches of this circular artery extend toward the pupil to supply the radially-oriented capillary net of the iris. They are characterized by undulation and form a radiating vascular net in the iris (Fig. 2A, B). The venules also follow an undulating course and empty into the scleral venous plexus (the venous circle of Hovius) via the pars plana venules. Smaller branches also originate from the arterial circle and travel towards the sclerocorneal junction (Figs. 3, 4).

The third palpebra and bulbar conjunctiva are supplied by

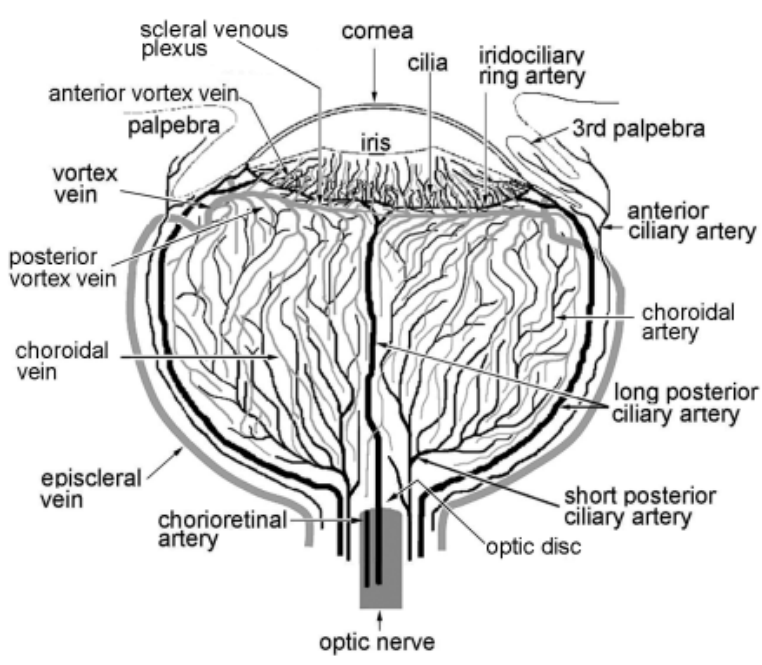

Fig. 1. Diagrammatic representation of the bulbar vessels of the rabbit eye.

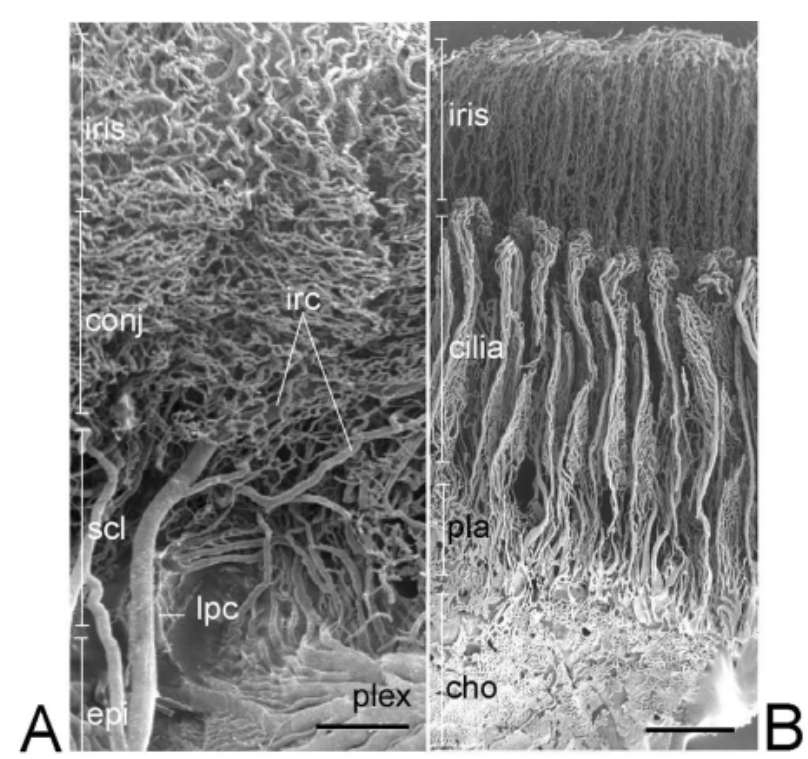

Fig. 2A. Scanning electron micrograph montage of the iris and bulbar conjunctiva (conj): view from outside the eye. epi: episcleral region; irc: iridociliary ring artery, lpc: long posterior ciliary artery; plex: sclera venous plexus; scl: sclerocorneal junction. $\mathrm{Bar}=500 \mu \mathrm{m}$.

Fig. 2B. Scanning electron micrograph montage of the vasculature of the iris and ciliary process (cilia): view from inside the eye. Note the radially arranged capillaries merging into the venules in the pars plana (pla). cho: choroid. Bar $=500 \mu \mathrm{m}$.

the anterior ciliary artery. The palpebral conjunctiva is fed by the palpebral artery, which diverges from the external carotid artery. The capillaries in the third palpebral (Fig. 5), bulbar (Fig. 6) and palpebral conjunctiva (Fig. 7) form a single-layered, relatively coarse capillary bed, near and approximately parallel to the basement membrane of the 


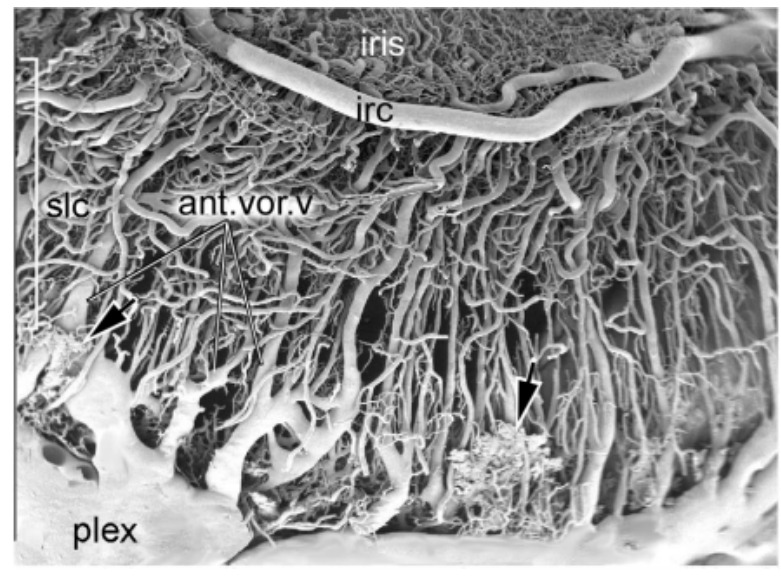

Fig. 3. Episcleral vasculature: view from outside the eye, showing the scleral venous plexus (the venous circle of Hovius (plex)), with which the anterior vortex veins (ant.vor.v) merge. Capillaries in the conjunctiva have been removed to show the anterior vortex veins. Note the trabecular reticulum (arrows). irc: iridociliary ring artery; scl: sclerocorneal junction. Bar $=500 \mu \mathrm{m}$.

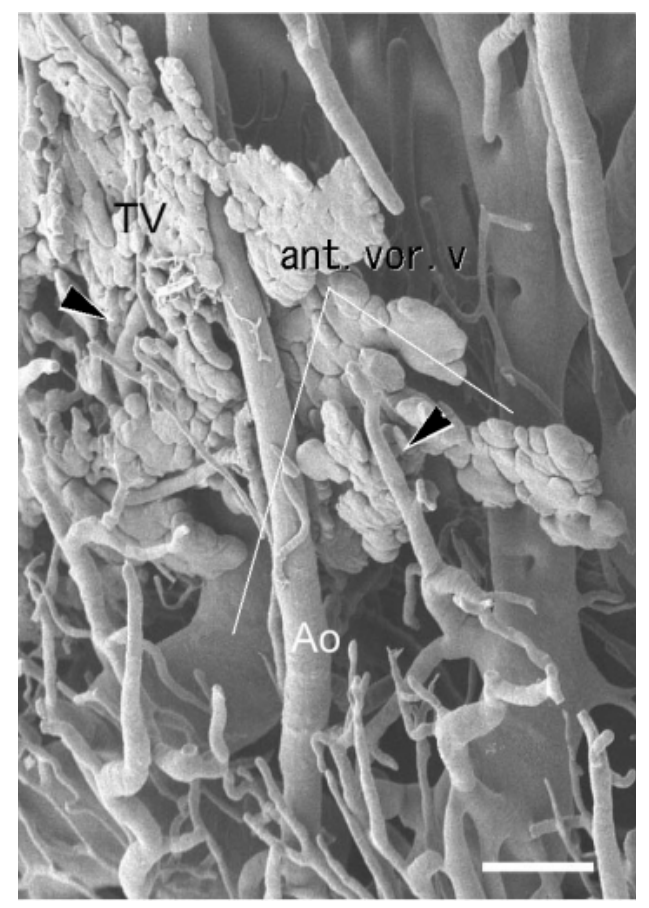

Fig. 4. Enlargement of area indicated by white arrows in Fig. 3, showing the trabeculae (TV) and an afferent channel (arrowheads) to the Hovius circle, the anterior vortex veins (ant.vor.v) and arterioles (Ao) from the iridociliary ring artery. Bar $=100 \mu \mathrm{m}$.

epithelium. The capillaries in the bulbar conjunctiva form a relatively compact capillary bed with short hairpin loops (Fig. 6). The venules draining the palpebral and conjunctival capillaries are disposed deeper in the stroma and form a well developed venous plexus underneath the capillary net- work. These venules are in turn drained by the anterior ciliary veins.

Vasculature of the posterior segment of the eyeball: The blood supply of the retina originates from the chorioretinal artery (Fig. 1). This artery divides into two retinal arteries just before the optic disc. The retinal arteries travel in horizontal bands on either side of the optic disc and pursue a wavy course to the base of the ciliary body, forming a merangiotic vasculature in which the retinal vessels extend partially to the retina (Figs. 8,9). The precapillary arterioles follow a relatively long course and then expand into capillaries. At their branching sites, the retinal precapillary arterioles show luminal constrictions, known as "intra-arterial cushions". The retinal capillaries are thin (8.0-8.9 $\mu \mathrm{m}$ in diameter) and form a thin capillary net layer in the superficial region (vitreous side) of the retina (Fig. 10). They drain into the postcapillary venules, which extend into a venous network just under the retinal capillary network. The venules then travel upward to drain into larger veins, which merge into the posterior ciliary vein via the retinal vein near the optic disc.

The short posterior ciliary arteries branch into the choroidal arteries, which run toward the anterior eye segments and supply the entire choroid (Fig. 1). All of the choroidal arteries are relatively flat and run in parallel arrays so as to interdigitate with the choroidal veins draining this region. The arteries ramify two or three times into precapillary arterioles. These choroidal precapillary arterioles are characterized by a very short course to the choriocapillaris, contrary to the characteristics of the retinal arterioles. Intra-arterial cushions were observed in the precapillary arterioles in the choroids, as well as in the retina. Choroidal capillaries are somewhat flat, sinusoid-like vessels with a luminal diameter ranging from $16.1-23.6 \mu \mathrm{m}$, providing a large surface area for metabolic exchange with the retinal pigment epithelium. Regional variations in choriocapillary morphology are well recognized; the capillary network is densely packed at the avascular region with a high capillary-to-intercapillary ratio. As with their corresponding arterioles, the venules collecting blood from the choriocapillaris also show a very short course. These venules then enlarge to a diameter of about $40 \mu \mathrm{m}$. The choroidal veins travel anteriorly, run parallel to the arteries and follow the curvature of the eyeball in the choroid layer, and then coalesce into the posterior vortex vein near the sclerocorneal junction.

Venous drainage of the eyeball: The venous blood from the iris and ciliary process drains into the episcleral venous plexus around the sclerocorneal junction via the venules of the pars plana (Fig. 2B). The episcleral venous plexus forms a fine, radially-oriented, extensive network (Fig. 3). The venous plexus then drains into the anterior segment of the vortex vein, known as the scleral venous plexus (Figs. 2A, 3). Finally, it drains into the vortex veins together with the posterior segment of the vortex vein, which drains blood from the entire choroid. There are 4 vortex veins, located obliquely on the dorsal, ventral, nasal, and temporal sides of the eyeball near the sclerocorneal junction. The vortex 


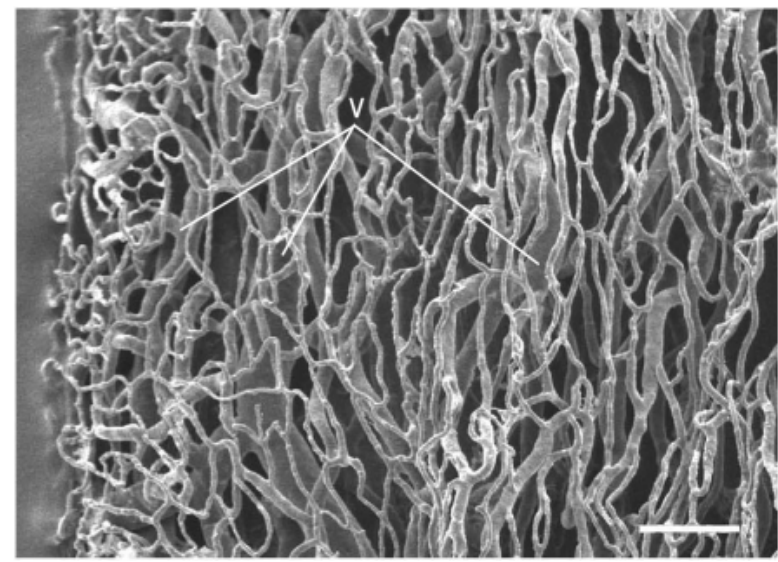

Fig. 5. Capillaries in the third palpebra. This capillary network is relatively sparse compared to that in the bulbar conjunctiva (Fig. 6) and palpebral conjunctiva (Fig. 7). The well developed venous plexus (v) is seen just under the capillary network. Bar $=100 \mu \mathrm{m}$.

veins are broad and quite flat. They empty into the episcleral veins following the curvature of the eyeball in the orbital fossa. The posterior ciliary vein of the rabbit is a small vein draining blood only from the retina.

Drainage of aqueous humor: The vessels for aqueous drainage are filled with acrylic resin, indicating inflow into the trabecular meshwork by retrograde from the scleral venous plexus (Figs. 3, 4). The trabecular reticulum was found to consist of tiny masses with a granular or fish scalelike appearance around the sclerocorneal junction. Numerous aqueous veins $(22.2-27.5 \mu \mathrm{m}$ in diameter, $1.4-3.0 \mathrm{~mm}$ in length) draining blood mixed with aqueous humor extend radially from the suprachoroidal space, communicating with the scleral venous plexus via the episcleral venous plexus.

\section{DISCUSSION}

The microvasculature in the third palpebral, bulbar and palpebral conjunctiva is characterized by a compact, singlelayered capillary network just under the epithelium and a rich venous plexus in the stroma. Such a subepithelial capillary network may play a role in the secretion of aqueous humor to prevent the cornea from drying, and in the exchange of nutrients and gasses between the cornea and the palpebral vessels across the palpebral epithelium when the lids are shut during sleep [15]. The well developed venous plexus just under the epithelium may be responsible for the ready absorption of eye drops and the longer lingering of eye drops in the conjunctiva than in the iris, cilia, retina and choroids [16].

The arterioles and venules of the iris are arranged radially and exhibit a round, uniform bore and an undulating or somewhat coiled shape, presumably to accommodate changes in the state of the pupil.

The ciliary vasculature, with separate plates of vessels arranged concentrically, is presumably arranged as such in order to facilitate the dilation and shrinkage of the eyeball to

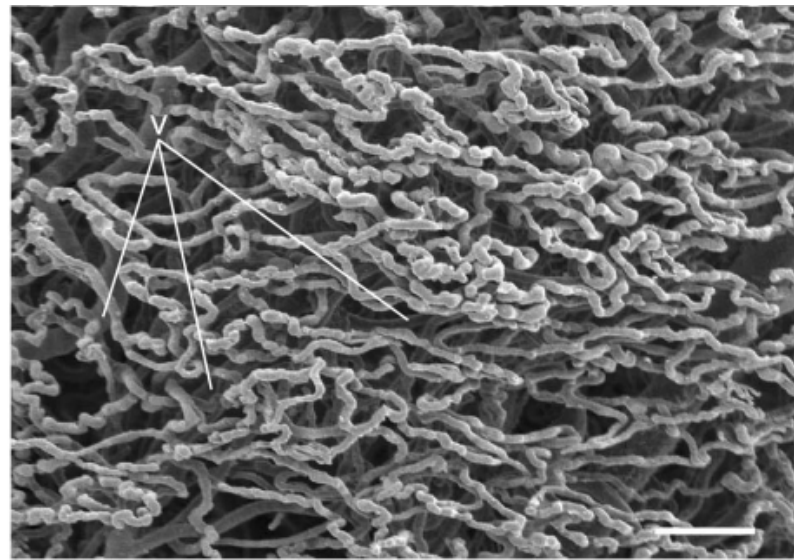

Fig. 6. Capillaries in the bulbar conjunctiva. Note the dense capillary bed with low hairpin loops just under the epithelium and the well developed venous plexus (v). Bar $=100 \mu \mathrm{m}$.

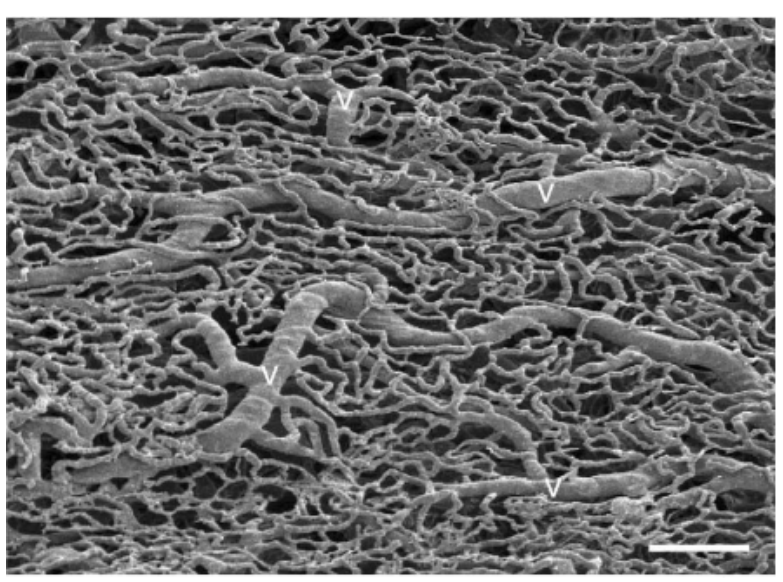

Fig. 7. Capillaries in the palpebral conjunctiva. Note the profuse venous plexus (v) and thin, single-layered capillary network. $\mathrm{Bar}=100 \mu \mathrm{m}$.

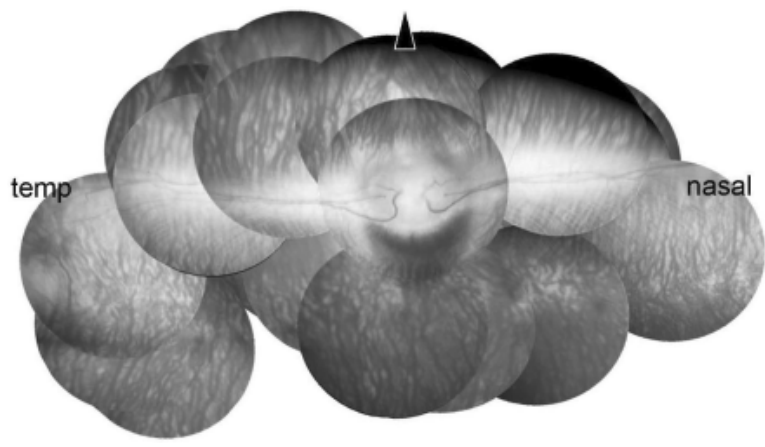

Fig. 8. Ophthalmic photograph of the right eye of an adult rabbit showing the horizontal spread of the retinal vessels. Arrowhead: 12 o'clock. nasal: nasal side; temp: temporal side.

accommodate variations in intraocular pressure. The capillaries supplying the anterior margin of the ciliary process exhibit a thick, irregular bore and form thoroughfare chan- 


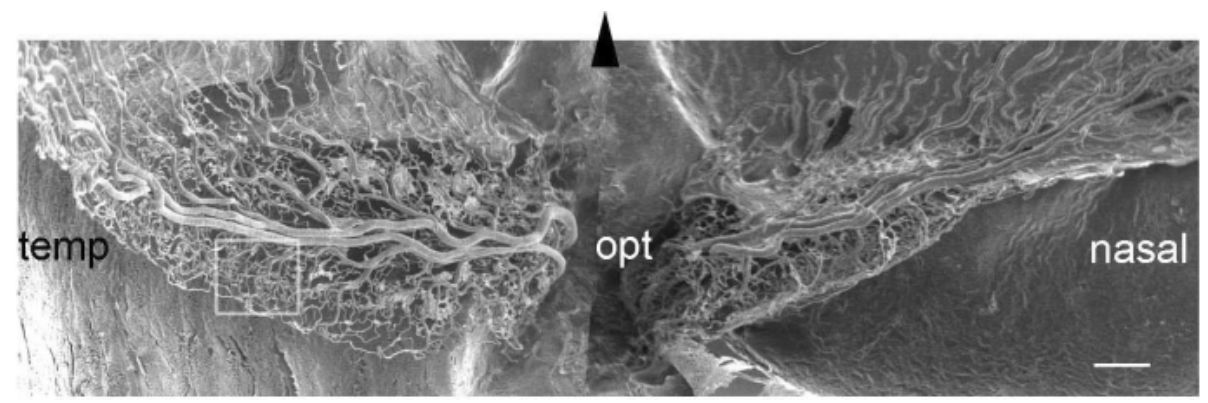

Fig. 9. Scanning electron micrograph montage of the entire retinal vasculature of the right eye in the rabbit. The rabbit's retinal vasculature is merangiotic, meaning that a compact network extends horizontally and partially in the retina. Casting orientation conforms to the orientation of Fig. 8. Arrowhead: 12 o'clock. nasal: nasal side; temp: temporal side; opt: optic disc. Bar=500 $\mu \mathrm{m}$.

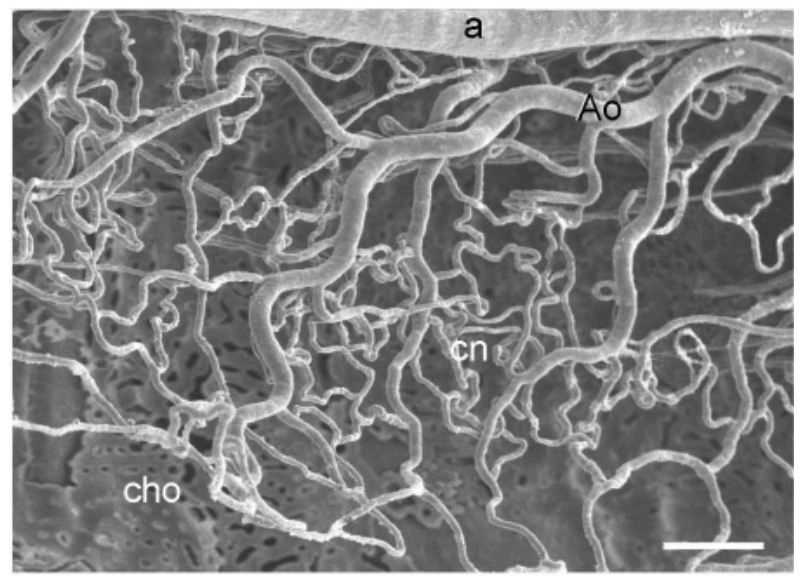

Fig. 10. Enlargement of area outlined in Fig. 9 showing retinal capillaries (cn). In the background is the choriocapillaris (cho). a: retinal artery; Ao: arteriole. Bar $=100 \mu \mathrm{m}$.

nels, draining blood from the ciliary arterioles into the pars plana venules, bypassing the ciliary processes entirely. This pattern of venous drainage is also seen in other species [11]. The thick marginal capillaries may be responsible for the very high blood flow values found in comparison to those of the choroid, kidney cortex and cardiac muscle [1], and for the blood flow velocities in the ciliary process [5]. The thoroughfare channels may be the equivalent of arteriovenous anastomoses, wherein the blood in the pars plana venules is not truly venous blood, but rather highly oxygenated blood [5].

The present SEM observation of the corrosion casts of the rabbit eye revealed the frequent occurrence of intra-arterial cushions at the branching sites in the retinal and choroidal arterioles, as seen in other organs [7]. The cushions are sphincter-like thickenings of the intimae and play a significant role in the regulation of blood flow at the branching sites $[7,13,14]$ and in causing plasma-skimming $[4,10,17]$. Plasma skimming has been reported to occur in the retinal vessels [10]. This phenomenon is characterized by the blockage of red blood cells from entering the capillaries, so that only plasma passes through the capillary bed. The retinal vascular network is an unrecognized disturbance to the optical filtering properties of the eye. A photon has a 40
$50 \%$ chance of encountering one or more capillaries, before it reaches a photoreceptor [22]. Capillaries containing little red blood cells may allow a photon to pass through the capillaries, preserving the optimal properties of the photon. The rabbit retina, which is characterized by the regional absence of retinal vessels, may allow for the direct capture of photons, facilitating the production of a clear, sharp image in the dark. The visual streak is found mainly in ungulates, and lagomorphs $[19,26]$ and is positioned with its long axis on a horizontal plane above the optic disc. Like the fovea centralis, it contains the highest concentration of photoreceptors and ganglion cells, and is the area that produces the greatest visual acuity. In the rabbit, the visual streak overlaps with the avascular field. The presence of a wide horizontal avascular retina overlapping the visual streak located just under the optic disc would permit scanning a broad visual field without extensive eye movement, whereas the presence of the best field of vision in the upper retina probably indicates an adaptation to searching for food at the ground level.

Blood is supplied to the choroid via short precapillary arterioles with comparatively large diameters and thick, compactly arranged capillaries, whereas blood is supplied to the retina via long arterioles with narrow diameters and extremely thin capillaries, forming a relatively sparse network. The anatomical differences between the 2 vessels may indicate differences in the velocities of blood flowing to the choroid and retina, in which choroidal blood flows in relatively high amounts [3]. Indeed, upon angiographic inspection, the filling pattern of fluorescein in the eye was successively observed during the choroidal phase, which is characterized by the initial filling of the choriocapillaris, and the retinal arterial phase, which in turn is characterized by the hyperfluorescence of the retinal arteries [3]. Again, the rich supply of blood, with high oxygen pressure and high vascular permeability, to the choriocapillaris would be expected considering the role of the choriocapillaris in supplying oxygen and nutrients to the retina. Such rich choroidal vasculature compensates for the decrease in arterial blood pressure and thereby remains relatively stable within a physiological range of arterial blood pressure [18]. As the retina receives oxygen and nutrients from the retinal and 
choroidal vessels, the rich choriocapillaris may compensate for the absence of retinal vessels for retinal nourishment, even though avascular retinas have the potential for anaerobic metabolism [28].

The iris, the cilia and the entire choroid of the rabbit are drained only by the vortex veins. In contrast, the rat eye has two drainage pathways; the posterior choroid is drained by the posterior ciliary vein, and the anterior segment of the eyeball is drained by the vortex veins [2, 13]. Rats have been used exclusively in the past in order to study the elevation in intraocular pressure triggered by the cauterization of vortex veins. However, the rats in these studies showed no elevation in intraocular pressure $[6,9]$. Presumably, additional venous drainage via the posterior ciliary vein compensated for the cauterization of the vortex veins to prevent intraocular pressure from increasing. The rabbit has only one major pathway for drainage of blood from the eyeball, as does the hamster [14], and one may successfully induce an increase in intraocular pressure by cauterizing its vortex veins. The rabbit could be a suitable animal model for the more efficient study of the etiology, pathogenesis, diagnosis and therapy of eye diseases such as glaucoma, which are caused by elevated intraocular pressure.

The aqueous humor exits the eye through the trabecular reticulum in the anterior chamber and merges into the venous plexus of the vortex system, known as the scleral venous plexus. The venous plexus also performs iridal and choroidal venous drainage and drains blood mixed with aqueous humor into the vortex veins. The aqueous drainage of the rabbit follows the same route as those of the dog [25], cat [27] and horse [21]. These animals are distinguishable from rats [12], primates [24] and humans [3] in that they lack a scleral venous sinus.

\section{REFERENCES}

1. Alm, A. 1983. The Physiology and Pharmacology of the Microcirculation. Academic Press, New York.

2. Bhutto, I. A. and Amemiya, T. 2001. Microvascular architecture of the rat choroid: corrosion cast study. Anat. Rec. 264: 63-71.

3. Duke-Elder, S. and Gloster, J. 1968. System of Ophthalmology. Volume C. Henry Kimpton, London.

4. Fourman, J. and Morfat, D. B. 1961. The effect of intra-arterial cushions on plasma skimming in small arteries. J. Physiol. 158: 374-380.

5. Funk, R. H., Wagner, E. and Wild, J. 1992. Microendoscopic observations of the hemodynamics in the rabbit ciliary processes. Curr. Eye Res. 11: 543-551.

6. Grozdanic, S. D., Betts, D. M., Sakaguchi, D. S., Kwon, Y. H., Kardon, R. H. and Sonea, I. M. 2003. Temporary elevation of the intraocular pressure by cauterization of vortex and episcleral veins in rats causes functional deficits in the retinal and optic nerve. Exp. Eye Res. 77: 27-33.

7. Heidger, P.M. Jr., Van Orden, D. E. and Fariey, D. B. 1983. Electron microscopic and histochemical characterization of intra-arterial cushions of the rat and porcine uterine vascular bed. Acta Anat. 117: 239-247.

8. Hunold, W., Wilmanns, I. and Freitag, U. 1978. Spatial arrangement and inner wall surface of ocular vessels. Scanning elec- tronmicroscopy of vascular casts. Albrecht Von Graefes. Arch. Klin. Exp. Opthalmol. 206: 71-78 (in German).

9. Kanamori, A., Nakamura, M., Mukuno, H., Maeda, H. and Negi, A. 2004. Diabetes has an additive effect on neural apoptosis in rat retina with chronically elevated intraocular pressure. Curr. Eye Res. 28: 47-54.

10. Lemmingson, W. 1971. Changes in the pattern of retinal blood circulation caused by plasma skimming. Klin. Monatsbl. Augenheilkd. 159: 790-793 (in German).

11. Morrison, J. C., Defrank, M. P. and Buskirk, M. V. 1987. Comparative microvascular anatomy of mammalian ciliary process. Invest. Ophthalmol. Vis. Sci. 28: 1325-1340.

12. Morrison, J. C, Fraunfelder, F. W., Milne, S. T. and Moore, C. G. 1995. Limbal microvasculature of the rat eye. Invest. Ophthalmol. Vis. Sci. 36: 751-756.

13. Ninomiya, H. and Kuno, H. 2001. Microvasculature of the rat eye: Scanning electron microscopy of vascular corrosion casts. Vet. Ophthalmol. 4: 55-59.

14. Ninomiya, H. and Inomata, T. 2005. Microvasculature of the hamster eye: scanning electron microscopy of vascular corrosion casts. Vet. Ophthalmol. 8: 7-12.

15. Oduntan, A. O. 1992. Organization of capillaries in the primate conjunctiva. Ophthalmic. Res. 24: 40-44.

16. Ooishi, M., Oomomo, A., Sakaue, F., Tazawa, H., Miyao, M., Kuriyama, H., Ikejiri, Y. and Sameshima, S. 1988. Studies on intraocular penetratin of NY-198 (Lomefloxacin) eye drops. Jpn. J. Ophthalmol. 92: 103-110 (in Japanese with English summary).

17. Perkkio, J., Wurzinger, L. J. and Schmid-Schonbein, H. 1987. Plasma and platelet skimming at T-junctions. Thromb. Res. 145: 517-526.

18. Reiner, A., Zagvazdin, Y. and Fitzgerald, M. E. 2003. Choroidal blood flow in pigeons compensates for decreases in arterial blood pressure. Exp. Eye Res. 76: 273-282.

19. Schwassmann, H. O. 2005. Aspect of retinotectal topography: Tectal magnification of specialized retinal areas and a possible center for binocular input. J. Exp. Zool. 256: 117-120.

20. Sheppard, L. B. 1959. Intrascleral drainage channels of the normal rabbit eye. Trans. Am. Ophthalmol. Soc. 57: 99-108.

21. Smith, P., Samuelson, D. and Brooks, D. 1988. Aqueous drainage paths in the equine eye: Scanning electron microscopy of corrosion cast. J. Morphol. 198: 33-42.

22. Snodderly, D. M. and Weinhaus, R. S. 1990. Retinal vasculature of the fovea of the squirrel monkey, Saimiri sciureus: threedimensional architecture, visual screening, and relationships to the neuronal layers. J. Com. Neurol. 297: 145-163.

23. Sugiyama, K., Bacon, D., Morrison, J. and Van Buskirk, M. 1992: Optic nerve head microvasculature of the rabbit eye. Invest. Ophthalmol. Vis. Sci. 33: 2251-2261.

24. Ujiie, K. and Bill, A. 1984. The drainage routes for aqueous humor in monkeys as revealed by scanning electron microscopy of corrosion casts. Scan. Electron. Microsc. 2: 849-859.

25. Van Buskirk, E. M. 1979. The canine eye: the vessels of aqueous drainage. Invest. Ophthamol. Vis. Sci. 18: 223-301.

26. Vaney, D. I. 1980. A quantitative comparison between the ganglion cell populations and axonal outflows of the visual streak and periphery of rabbit retina. J. Comp. Neurol. 189: 215-233.

27. Wong, V. G. and Macri, F. J. 1964. Vasculature of the cat eye. Arch. Ophthalmol. 72: 351-358.

28. Yu, D. Y. and Cringle, S. J. 2001. Oxygen distribution and consumption within the retina in vascularised and avascular retinas and in animal models of retinal disease. Prog. Retin. Eye Res. 20: $175-208$. 